\title{
Forecast of Stock Index Volatility Using Grey GARCH-Type Models
}

\author{
Li-Yan Geng ${ }^{1, *}$ and Zhan-Fu Zhang ${ }^{2}$ \\ ${ }^{1}$ School of Economics and Management, Shijiazhuang Tiedao University, Shijiazhuang, 050043, China; ${ }^{2}$ Department of \\ Electrical and Engineering, Sifang College, Shijiazhuang Tiedao University, Shijiazhuang, 051132, China
}

\begin{abstract}
This paper integrated the genetic algorithm (GA) and grey forecasting $(\mathrm{GM}(1,1))$ model into three GARCH-type models and proposed the GAGM-GARCH-type models. The GM $(1,1)$ model was used to modify the error terms of the GARCH-type models to improve the volatility forecasting performance of the traditional GARCHtype models. Meanwhile, as for the shortcomings in parameters estimation of GM $(1,1)$ model, the GA was adopted to find the optimal grey parameters of $\operatorname{GM}(1,1)$ model. Using the stock data of China stock market, the paper compared the performance of the GAGAM-GARCH-type models in out-of-sample volatility forecasting with those of the GM-GARCH-type, RGM-GARCH-type, and GARCH-type models. It is indicated by values of the evaluation criteria that the GAGM-GARCH-type models have better volatility forecasting performances relative to the other three types of GARCH-type models.
\end{abstract}

Keywords: Genetic algorithm, Grey GARCH-type models, Volatility forecasting, ARCH model, GM-GARCH-Type Models, Optimal grey parameters.

\section{INTRODUCTION}

Volatility is one of the important variables in financial economy study. Investment portfolio, asset pricing, risk management and monetary policy formulating, all depend on volatility. Therefore, it is necessary and important to model and forecast volatility of the financial market. To date, there are various models to analyze and forecast financial volatility. Among them, GARCH-type models developed from ARCH model [1] are more popular than the other types of volatility models. Furthermore, the three GARCH-type models: GARCH [2], EGARCH [3], and GJR-GARCH models [4], are widely used by researchers in modeling and forecasting volatility.

Financial time series usually contains known and unknown information due to the complexity of financial market. So, it is difficult for the traditional GARCH-type models to describe the unknown information in error terms sequence. Grey forecasting (GM $(1,1)$ ) model proposed by Deng is mainly used for a system with the uncertain information [5]. It shows advantages such as high short-term forecasting precision, less samples, and simple calculation [6]. Tseng used the forecasting property of GM $(1,1)$ model to modify the error terms of GARCH model and proposed GM-GARCH model [7]. Later, Tseng and Wang provided GM-EGARCH [8] and GM-GJR-GARCH models [9], utilizing GM $(1,1)$ model to modify the error terms of EGARCH and GJR-GARCH models. The results indicated that the introduction of GM $(1,1)$ model improved the short-term forecasting accuracy of the GARCH-type models to a certain degree. However, due to the theoretical shortcomings, GM $(1,1)$ model may produce larger forecast error when fore

*Address correspondence to this author at the 17\# East Road, Second North Ring, Shijiazhuang, China. Postcard: 050043; Tel: 15226509410; E-mail: gengliyan_28117@163.com casting the error sequences which are highly volatile. Genetic algorithm (GA) suggested by Holland is a powerful optimization algorithm and has been widely applied to various optimization problems. With the advantages of selforganizing and self-adaption, GA can find the global-optimal solution without trapping in the local-optimal points. Based on these characteristics, it was indicated that GA can enhance the forecasting accuracy of GM $(1,1)$ model $[10,11]$.

This paper used GA to estimate the grey parameters of GM $(1,1)$ model to increase the accuracy in error sequences forecasting, and then improved the forecasting performance of the GARCH-type models. The main structure of this paper is arranged as follows. Section 1 introduces the research background and objective of this paper. Section 2 summarizes the theory of GARCH-type and GM-GARCH-type models. Section 3 describes the theory of GA briefly and designs the procedure of GA-based parameter optimization for GM $(1,1)$ model. The empirical research on two stocks in China stock market is presented in Section 4. Finally, the conclusions and suggestions are provided in Section 5.

\section{GREY GARCH-TYPE MODELS}

\subsection{GARCH-Type Models}

Generalized autoregressive conditional heteroskedasticity (GARCH) model introduced by Bollerslev is the extension of ARCH model, which assumes that the current conditional variance is associated with the past conditional variances and the past random error. GARCH $(p, q)$ model with Gaussian disturbance can be expressed as:

$$
\begin{aligned}
& \varepsilon_{t}=\sigma_{t} v_{t} \quad N(0,1) \\
& \sigma_{t}^{2}=\omega+\sum_{i=1}^{p} \alpha_{i} \varepsilon_{t-i}^{2}+\sum_{j=1}^{q} \beta_{j} \sigma_{t-j}^{2}
\end{aligned}
$$


Where, $\omega$ denotes the uncertainty of the conditional variance. And $\alpha_{i}, \beta_{j}$ denote the short-term and long-term influence on the conditional variances, respectively. These parameters should satisfy the restrictions:

$$
\begin{aligned}
& \sum_{i=1}^{q} \alpha_{i}+\sum_{j=1}^{p} \beta_{j}<1 \\
& \omega, \alpha_{i}, \beta_{j} \geq 0
\end{aligned}
$$

The GARCH model has the ability of describing the phenomenon of volatility clustering and the distribution of fat tail existing in the financial assets returns, but it cannot explain the asymmetric features of the returns.

Nelson proposed exponential GARCH (EGARCH) model to capture the leverage effects of the assets price varying on conditional variance by adding an asymmetric term into the GARCH model. Different from GARCH model, EGARCH model defines the conditional variance as the logarithm form, which has no restrictions on the parameters $\alpha_{i}$ and $\beta_{j}$. The conditional variance equation of EGARCH $(p, q)$ is written as:

$$
\ln \sigma_{t}^{2}=\omega+\sum_{i=1}^{p}\left(\alpha_{i}\left|\frac{\varepsilon_{t-i}}{\sigma_{t-i}}-\sqrt{\frac{2}{\pi}}\right|+\gamma_{i} \frac{\varepsilon_{t-i}}{\sigma_{t-i}}\right)+\sum_{j=1}^{q} \beta_{j} \ln \sigma_{t-j}^{2}
$$

Where, the parameter $\gamma_{i}$ reflects the asymmetry of the returns. $\gamma_{i}>0$ represents that the positive return has a bigger impact on the volatility, $\gamma_{i}<0$ represents the negative return having bigger impact on the volatility and that there is no asymmetry when $\gamma_{i}=0$.

Glosten et al. added another asymmetric term into the GARCH model for account of the asymmetry of the return behavior. They called the proposed model as GJR-GARCH model. The conditional variance equation of GJR-GARCH $(p, q)$ is represented as:

$$
\sigma_{t}^{2}=\omega+\sum_{i=1}^{p}\left(\alpha_{i} \varepsilon_{t-i}^{2}+\gamma_{i} S_{t-i}^{-} \varepsilon_{t-i}^{2}\right)+\sum_{j=1}^{q} \beta_{j} \sigma_{t-j}^{2}
$$

Where, $S$ is a dummy variable. $S=1$ when $\varepsilon_{t-i}<0$, and $S=0$ when $\varepsilon_{t-i} \geq 0 . \gamma_{i}=0$ indicates no asymmetric effect, while $\gamma_{i} \neq 0$ indicates the presence of asymmetric effect. Additionally, the parameters of EGARCH model should meet the following restrictions:

$$
\begin{aligned}
& \sum_{i=1}^{p} \alpha_{i}+\sum_{j=1}^{q} \beta_{i}+\frac{1}{2} \sum_{j=1}^{p} \gamma_{i}<1 \\
& \omega, \alpha_{i}, \beta_{j} \geq 0, \alpha_{i}+\gamma_{i} \geq 0
\end{aligned}
$$

\subsection{GM-GARCH-Type Models}

According to the GARCH-type models, the current conditional variance $\sigma_{t}^{2}$ essentially depends on the past error terms $\varepsilon_{\tau}(\tau<t)$, but this is not consistent with the actual situation. In the actual financial market, with the exclusion of the past price, the error terms are also affected by the uncertain factors, such as the economic, political, environmental and other complex factors. These factors cause the changing of errors all the time. Thus, the current error may have an impact on the current conditional variance $\sigma_{t}^{2}$, while the traditional GARCH-type models may just neglect this point.

Grey forecasting model is the core model of the grey system theory. Using the accumulated generating operation (AGO) to preprocess the original data, grey forecasting model finds and grasps the development law of the system, and then forecasts the future state of the system quantitatively. GM $(1,1)$ model is the commonly used grey forecasting model.

To strengthen the impact of the current error on the current conditional variance, GM $(1,1)$ model was used to continuously modify the squared error term sequences of the GARCH-type models (GM-GARCH-type models). That is, the one-step-ahead forecasted error values obtained from GM $(1,1)$ model were put into the conditional variance equations for enhancing the forecasting ability of the GARCHtype models, as shown in the literature [7-9]. The conditional variance equations of the GM-GARCH-type models are written as:

$$
\begin{aligned}
& \sigma_{t}^{2}=\omega+\sum_{i=1}^{p} \alpha_{i}\left(\varepsilon_{t-i}^{2}+\hat{\varepsilon}_{t}^{2}\right)+\sum_{j=1}^{q} \beta_{j} \sigma_{t-j}^{2} \\
& \ln \sigma_{t}^{2}=\omega+\sum_{i=1}^{p}\left(\alpha_{i}\left|\frac{\varepsilon_{t-i}+\hat{\varepsilon}_{t}}{\sigma_{t-i}}-\sqrt{\frac{2}{\pi}}\right|+\gamma_{i} \frac{\varepsilon_{t-i}+\hat{\varepsilon}_{t}}{\sigma_{t-i}}\right)+\sum_{j=1}^{q} \beta_{j} \ln \sigma_{t-j}^{2} \\
& \sigma_{t}^{2}=\omega+\sum_{i=1}^{p}\left[\alpha_{i}\left(\varepsilon_{t-i}^{2}+\hat{\varepsilon}_{t}\right)+\gamma_{i} S_{t-i}^{-}\left(\varepsilon_{t-i}^{2}+\hat{\varepsilon}_{t}\right)\right]+\sum_{j=1}^{q} \beta_{j} \sigma_{t-j}^{2}
\end{aligned}
$$

Where, $\hat{\varepsilon}_{t}$ represents the random error forecasts obtained by using the GM $(1,1)$ model.

\section{GM-GARCH-TYPE MODELS WITH GA}

\subsection{Genetic Algorithm}

Genetic algorithm (GA) is a global optimization algorithm, following the mechanics of biological evolution. It mimics the phenomena of reproduction, mating and mutation which occur in the process of natural selection and natural inheritance. Based on the natural law of survival of the fittest, GA produces the preferred individual generation by generation and finds the optimal individual by using the genetic operators such as selection, crossover and mutation. The details on the genetic operators can be found in the literature [12].

GA has its own characteristics apart from all the features of evolutionary computation: (1) GA directly deals with the code set of the decision variables rather than the actual value itself. During the search process, GA neither places any constraints on the continuity of the optimized function nor requires existence of the derivative of the optimized function. (2) GA looks for optimal solution by using multi-point search or group search, which shows high implicit parallel- 
ism. (3) GA is an adaptive search technique. The selection, crossover and mutation are operated in a probabilistic manner, increasing the flexibility of the search process. At the same time, GA can converge to the optimal solution with a large probability. Accordingly, GA shows good ability in the global search and optimization. (4) GA has better general adaptability and extensibility since GA takes the objective function value as the search information without requirement regarding the behavior of the function. Meanwhile, GA improves the search efficiency by allowing users to concentrate on the higher fitness degree from the search range to the search space.

\subsection{The Optimal Grey Parameters by GA}

Grey parameters $a$ and $b$ are important for the $\operatorname{GM}(1,1)$ model. The forecasting performance of the GM $(1,1)$ model depends on the accuracy of the parameters solution to $a$ and $b$. In GM $(1,1)$ model, the estimators of $a$ and $b$ are obtained by the least squares method under the assumption that the random error sequences are normally distributed. However, due to being affected by various complex factors, the error sequences do not follow the normality distribution. As a result, the parameter estimators by the least squares method may be biased and non-consistent. Moreover, when estimating the two parameters, the least squares method should satisfy the restriction of $\hat{x}^{(1)}(1)=x^{(1)}(1)=x^{(0)}(1)$, which may cause larger system error and impact on the forecasting accuracy of GM $(1,1)$ model. To improve the forecasting ability of GM $(1,1)$ model in error terms sequence forecasting, GA is applied to search for the optimal grey parameters of GM $(1,1)$ model. The general procedure of GA-based parameter optimization to GM $(1,1)$ model for forecasting the error sequences can be summarized as follows:

Step 1: Preprocessing the data. Transform the original error terms sequence $\varepsilon^{(0)}=\left(\varepsilon^{(0)}(1), \varepsilon^{(0)}(2), \ldots, \varepsilon^{(0)}(n)\right)$ with $\varepsilon^{(0)}(t) \in R, \quad t=1,2, \ldots, n$ into the non-negative sequence $u^{(0)}=\left\{u^{(0)}(1), u^{(0)}(2), \cdots, u^{(0)}(n)\right\}$, where

$u^{(0)}(t)=\varepsilon^{(0)}(t)+\min \left(\varepsilon^{(0)}(t)\right), t=1,2, \ldots, n$.

Step 2: Initialization population. Initialize the parameters of GA, consisting of the population size, the number of evolutionary generation, the crossover rate and mutation rate.

Step 3: Definition objective function. The objective function is defined based on the criterion of minimizing the mean squares error:

$$
F=\frac{1}{1 / n \sum_{t=1}^{n}\left(\hat{u}_{t}^{(0)}(t)-u_{t}^{(0)}(t)\right)^{2}}
$$

Where $\hat{u}_{t}^{(0)}$ and $u_{t}^{(0)}$ are the forecasted and actual error values, respectively.

Step 4: Evolution operation. Calculate the objective value of each individual in the population and search for the optimal solution by the steps of selection, crossover, mutation and evolution.

Step 5: Evolution stops. Repeat Step 3 to Step 4 until the number of evolutionary generation is met, when the optimal grey parameters $a^{*}$ and $b^{*}$ are obtained.

Step 6: Model construction and forecasting. Using the obtained parameters $a^{*}$ and $b^{*}$, the GA-based GM $(1,1)$ model (called GAGM $(1,1)$ ) is constructed as:

$$
\hat{u}^{(1)}(t)=\left(u^{(0)}(1)-\frac{b^{*}}{a^{*}}\right) e^{-a^{*}(t-1)}+\frac{b^{*}}{a^{*}}, t=2, \ldots, n .
$$

Then, the error values forecasted by GAGM $(1,1)$ model are transformed into the original error forecasts:

$$
\hat{\varepsilon}^{(0)}(t)=\left(1-e^{a^{*}}\right)\left(\varepsilon^{(0)}(1)-\frac{b^{*}}{a^{*}}\right) e^{-a^{*}(t-1)}-\min \left(\varepsilon^{(0)}(t)\right) t=2, \ldots, n .(15)
$$

Finally, $\hat{\varepsilon}^{(0)}(t)$ is added in the conditional variance equations of the GARCH-type models.

\section{EMPIRICAL RESEARCH}

\subsection{Data Description}

Two stock indices of China stock market were examined: HuShen 300 Index (HS300) and HangSeng Index (HSI). The daily trading prices of the two stock indices were extracted from Sina website during July 4, 2011 to July 10, 2014, including 1338 observations. The continuously compounded logarithmic returns were calculated by using the daily closing prices: $r_{t}=\ln P_{t}-\ln P_{t-1}$, Where $P_{t,}, P_{t-1}$ are the daily closing prices for day $t$ and $t$-1, respectively. The descriptive statistics of the daily return series of HS300 and SZCI can be found in Table 1.

It is clear from Table $\mathbf{1}$ that for the two indices, the means of the return series are close to zero, significantly smaller than the corresponding standard deviation within the considered period. Thus, the conditional mean of the return

\begin{tabular}{|c|c|c|c|c|c|c|c|c|c|c|}
\hline Indices & Mean & Max. & Min. & Std. Dev. & Skewness & Kurtosis & J-B test & $\mathbf{L B}(\mathbf{2 0})$ & $\mathbf{L B}^{2}(\mathbf{2 0})$ & LM(20) \\
\hline HS300 & 0.0001 & 0.0646 & -0.0737 & 0.0151 & -0.2803 & 5.0937 & $261.7087^{\mathrm{ab}}$ & 17.2411 & $233.6812^{\mathrm{ab}}$ & $120.1993^{\mathrm{ab}}$ \\
\hline $\mathrm{SZCI}$ & 0.0005 & 0.0599 & -0.0741 & 0.0162 & -0.5474 & 4.4599 & $185.5093^{\mathrm{ab}}$ & $31.6339^{\mathrm{a}}$ & $250.2407^{\mathrm{ab}}$ & $127.4946^{\mathrm{ab}}$ \\
\hline
\end{tabular}
series can be assigned as zero. The J-B test of the return se-

Table 1. Descriptive statistics of daily return series of HS300 and SZCI.

Note: JB test is the Jarque-Bera normality test for the distribution of the return series. LB(20), LB2(20) are the Ljung-Box test for the $20^{\text {th }}$ order serial correlation of the return and squared return series, respectively. LM (20) is the Engle's (1982) LM test for heteroscedasticity of the return series. a, b denote significance at the $5 \%$ and $1 \%$ levels, respectively. 
ries rejects the null hypothesis of normality at $1 \%$ and $5 \%$ levels, respectively. Besides, negative Skewness and high Kurtosis being found in the return series, it is shown that the distribution of the return series is negatively biased and fattailed. $\mathrm{LB}(20)$ statistic for serial correlation suggests that the return series of SZCI has serial correlation at the 5\% significance level, while the return series of HS300 has no significant serial correlation. $\mathrm{LB}^{2}(20)$ and ARCH (20) tests support the rejection of the null hypothesis of heteroskedasticity at $1 \%$ and $5 \%$ levels, indicating that the strongly ARCH effect exists in the two return series. It is reasonable to construct the GARCH-type models.

\subsection{Empirical Results}

The daily returns of HS300 and SZCI, normalized in the range from 0 to 1 , are classified into two sections: the first 1000 daily returns are used for model training, and the remaining 337 ones for model evaluation. The rolling forecasting method is used to forecast the stochastic error of the GARCH-type models. When estimating the grey parameters of GM $(1,1)$ model, the control parameters of GA are set as: population $\operatorname{size}=30$; crossover rate $=0.95$; mutation rate $=0.08$, number of evolutionary generation $=50$. For HS300 and SZCI, GAGM $(1,1)$ model forecasts the next error value of the GARCH-type models using the eighteen most recent errors. The forecasted error values were added into the variance equation of the GARCH-type models and the parameters of the GARCH-type models were estimated by using the maximum likelihood estimation (QMLE) method. Then, the constructed GAGM-GARCH-type models were employed to one-step-ahead forecast volatility. To compare the forecasting results of the proposed models, three types of GARCH models were applied for forecasting volatility of HS300 and SZCI with the same data samples. They are GM-GARCHtype, RGM-GARCH-type, and GARCH-type models. Finally, the volatility forecasts were transformed into the original volatility forecasts.

The out-of-sample forecasting performances of each type of models were evaluated by four statistical indices: the root mean squared error (RMSE), the mean absolute error (MAE), the logarithmic error statistic (LL), and the Linear Exponential index (LINEX). These indices are defined by:

$$
\begin{aligned}
& \mathrm{RMSE}=\sqrt{T^{-1} \sum_{t=1}^{T}\left(\hat{\sigma}_{t}-R_{t}\right)^{2}} \\
& \mathrm{MAE}=T^{-1} \sum_{t=1}^{T}\left|\hat{\sigma}_{t}-R_{t}\right| \\
& \mathrm{LL}=T^{-1} \sum_{t=1}^{T}\left[\ln \left(\hat{\sigma}_{t}\right)-\ln \left(R_{t}\right)\right]^{2} \\
& \mathrm{LINEX}=T^{-1} \sum_{t=1}^{T}\left\{\exp \left[\chi\left(\hat{\sigma}_{t}-R_{t}\right)\right]-\chi\left(\hat{\sigma}_{t}-R_{t}\right)-1\right\}
\end{aligned}
$$

Where, $T$ is the number of volatility forecasts. $\hat{\sigma}_{t}$ is the square root of the volatility forecasts. $R_{t}$ is the proxy of the actual daily volatility. In this study, the range-based ex post volatility $R_{t}$ is taken as a proxy of the actual daily volatility, expressed as:
$R_{\mathrm{t}}=\sqrt{k}\left(\log \left(P_{t, h}\right)-\log \left(P_{t, l}\right)\right) \times 100$

Where, $P_{t, h}$ and $P_{t, l}$ are the intraday high and intraday low prices, respectively. $k$ is the calibration parameter between the range-based unconditional variance and the return-based unconditional variance. The four statistical indices measured the forecasting errors of the evaluated models. The model with smaller ones showed better volatility forecasting ability.

Table 2 lists the comparison of the results of the four types of models in forecasting volatility of HS300 and SZCI. It can be seen from Table 2: Firstly, for HS300 and SZCI, GAGM-GARCH-type models generate smaller RMSE, MAE, LL, and LINEX compared to the other types of models, which show a better performance than the other types of models in forecasting volatility. Among the three GAGMGARCH-type models, compared with GAGM-GJR-GARCH model, GAGM-GARCH model generates smaller RMSE and LINEX but larger MAE and LL, indicating that the forecasting ability of GAGM-GARCH model is somewhat mixed compared with that of the GAGM-GJR-GARCH model. GAGM-EGARCH model shows the worst volatility forecasting performance according to the four evaluation criteria. Secondly, with the exception of LINEX of RGM-GARCHtype models, GM-GARCH-type models produce smaller RMSE, MAE, and LL than RGM-GARCH-type and GARCH-type models, suggesting that on the whole, GMGARCH-type models outperform the RGM-GARCH-type and GARCH-type models in volatility forecasting. Thirdly, for HS300, RGM-GARCH-type models provide better volatility forecasting results in terms of RMSE, LL, and LINEX. While for SZCI, GARCH-type models seem to provide better volatility forecasting results in terms of RMSE, MAE, and LL. Hence, it is difficult to determine that which one of these two types of models is better in volatility forecasting performance.

The volatility forecasting results of the GAGM-GARCHtype models for HS300 and SZCI are shown in Figs. (1) and (2). As shown in the two figures, the three GAGMGARCH-type models can forecast the main volatility varying trend of the two stock indices, where GAGM-GARCH model provides superior volatility forecasts in the smaller fluctuation stage and GAGM-GJR-GARCH model provides superior volatility forecasts in the larger fluctuation stage.

\section{CONCLUSION}

To enhance the forecasting performance of the GARCHtype models, i.e., GARCH, EGARCH, and GJR-GARCH models, in this paper, GAGM-GARCH-type models were proposed, which combined genetic algorithm (GA) and $\operatorname{GM}(1,1)$ model with three GARCH-type models. $\operatorname{GM}(1,1)$ model optimized by GA was used to modify the error terms of the GARCH-type models. The results of the empirical study on HS300 and SZCI indices of China stock market show that the proposed models have superior performances in out-of-sample volatility forecasting than the GMGARCH-type, RGM-GARCH-type, and GARCH-type models. The GAGM-GARCH and GAGM-GJR-GARCH models perform better than the GAGM-EGARCH model, but the forecasting performance of the GAGM-GARCH model is 
Table 2. RMSE, MAE, LL, and LINEX of four types of volatility models for HS300 and SZCI.

\begin{tabular}{|c|c|c|c|c|c|}
\hline Indices & Models & RMSE & MAE & $\mathbf{L L}$ & LINEX \\
\hline \multirow{12}{*}{ HS300 } & GAGM-GARCH & 1.1042 & 0.7989 & 0.1599 & 0.0123 \\
\hline & GAGM-EGARCH & 1.1174 & 0.8054 & 0.1612 & 0.0163 \\
\hline & GAGM-GJR-GARCH & 1.1043 & 0.7968 & 0.1588 & 0.0127 \\
\hline & GM-GARCH & 1.2571 & 0.8164 & 0.1829 & 0.1167 \\
\hline & GM-EGARCH & 1.2710 & 0.8136 & 0.1829 & 0.1103 \\
\hline & GM-GJR-GARCH & 1.2650 & 0.8119 & 0.1811 & 0.0805 \\
\hline & RGM-GARCH & 1.5748 & 1.1962 & 0.3107 & 0.0555 \\
\hline & RGM-EGARCH & 1.4588 & 1.1316 & 0.2887 & 0.0593 \\
\hline & RGM-GJR-GARCH & 1.5534 & 1.1833 & 0.306 & 0.0347 \\
\hline & GARCH & 1.5868 & 1.0991 & 0.3776 & 0.9946 \\
\hline & EGARCH & 1.5926 & 1.1083 & 0.3847 & 0.9830 \\
\hline & GJR-GARCH & 1.5719 & 1.0879 & 0.368 & 0.6466 \\
\hline \multirow{12}{*}{$\mathrm{SZC}$} & GAGM-GARCH & 1.1219 & 0.8088 & 0.1545 & 0.1049 \\
\hline & GAGM-EGARCH & 1.1554 & 0.8247 & 0.1592 & 0.1256 \\
\hline & GAGM-GJR-GARCH & 1.1259 & 0.8079 & 0.1544 & 0.1150 \\
\hline & GM-GARCH & 1.3083 & 0.8534 & 0.1742 & 1.8603 \\
\hline & GM-EGARCH & 1.2958 & 0.8384 & 0.1678 & 1.7071 \\
\hline & GM-GJR-GARCH & 1.2798 & 0.8408 & 0.1677 & 0.9225 \\
\hline & RGM-GARCH & 1.7921 & 1.4416 & 0.3921 & 0.8802 \\
\hline & RGM-EGARCH & 1.3388 & 0.9899 & 0.3611 & 0.5100 \\
\hline & RGM-GJR-GARCH & 1.7536 & 1.4095 & 0.3803 & 0.6578 \\
\hline & GARCH & 1.632 & 1.1177 & 0.348 & 14.9011 \\
\hline & EGARCH & 1.6081 & 1.1012 & 0.3307 & 13.4074 \\
\hline & GJR-GARCH & 1.5914 & 1.0979 & 0.3267 & 6.789 \\
\hline
\end{tabular}

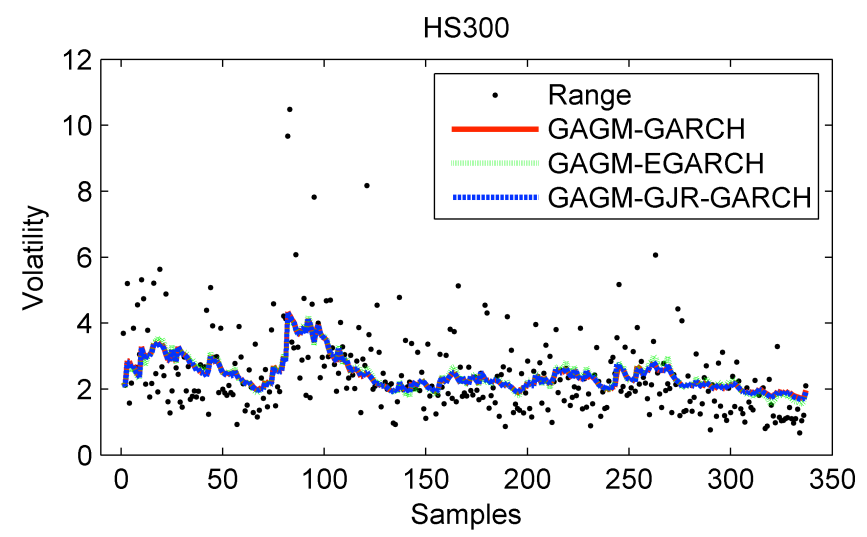

Fig. (1). HS300 volatility forecasts by GAGM-GARCH-type models.

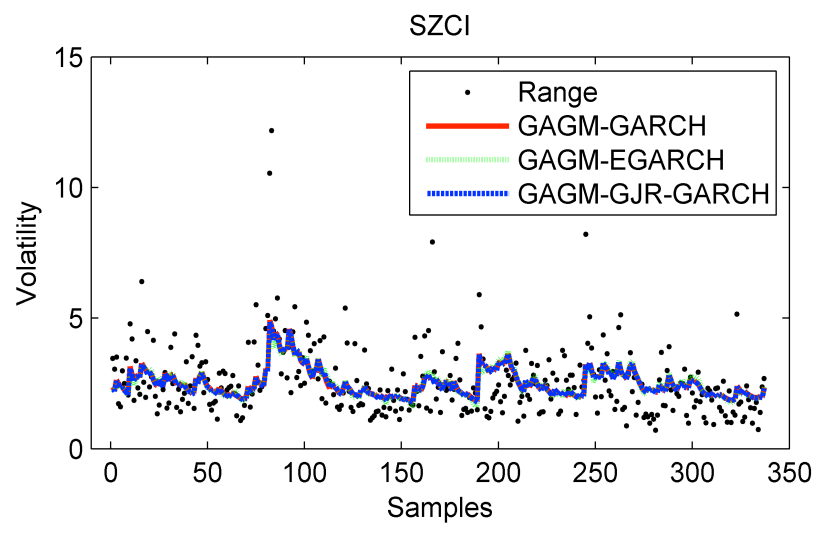

Fig. (2). SZCI volatility forecasts by GAGM-GARCH-type models. 
somewhat mixed compared to the GAGM-GJR-GARCH model. In addition, the GM-GARCH-type models, as a whole, produce superior volatility forecasts compared to the RGM-GARCH-type and GARCH-type models. While, the RGM-GARCH-type and GARCH-type models show different volatility forecasting abilities according to different evaluation criteria, which need to be further studied.

\section{CONFLICT OF INTEREST}

The authors confirm that this article content has no conflict of interest.

\section{ACKNOWLEDGEMENTS}

This work was supported by the Scientific Research Foundation of the Ministry of Education of China for Young Scholars "Intelligent Forecasting Methods for Financial Volatility and Its Empirical Research" (No. 11YJC790048).

\section{REFERENCES}

[1] R. F. Engle, "Autoregressive conditional heteroskedasticity with estimates of the variance of united kingdom inflation", Econometri$c a$, vol. 50, pp. 987-1007, July 1982 .

[2] T. Bollerslev, "Generalized autoregressive conditional heteroscedasticity", Journal of Econometrics, vol. 31, pp. 307-327, April 1986.
[3] D. Nelson, "Conditional heteroskedasticity in asset returns: A new approach", Econometrica, vol. 59, pp. 347-370, March 1991.

[4] L. R. Glosten, R. Jagannathan, and D. E. Runkle, "On the relation between the expected value and the volatility of the nominal excess return on stocks", Journal of Finance, vol. 48, pp. 1779-1801, December 1993.

[5] S. F. Liu and N. M. Xie, Grey System Theory and Its Applications. Science Press, Beijing, 2014.

[6] A. Samvedi and V. Jain, "A grey approach for forecasting in a supply chain during intermittent disruptions", Engineering Applications of Artificial Intelligence, vol. 26, pp. 1044-1051, March 2013.

[7] C. H. Tseng, S. T. Cheng and Y. H. Wang, "New hybrid methodology for stock volatility prediction", Expert Systems with Applications, vol. 36, pp. 1833-1839, March 2009.

[8] C. H. Tseng, S. T. Cheng, Y. H. Wang and J. T. Peng, "Artificial neural network model of the hybrid EGARCH volatility of the taiwan stock index option prices", Physica A: Statistical Mechanics and its Applications, vol. 387, pp. 3192-3200, January 2008.

[9] Y. H. Wang, "Nonlinear neural network forecasting model for stock index option Price: hybrid GJR-GARCH approach", Expert Systems with Applications, vol. 36, pp. 564-570, January 2009.

[10] Y. S. Lee and L. I. Tong, "Forecasting nonlinear time series of energy consumption using a hybrid dynamic model", Applied Energy, vol. 94, pp. 251-256, February 2012.

[11] L. C. Hsu, "A genetic algorithm based nonlinear grey bernoulli model for output forecasting in integrated circuit industry", Expert Systems with Applications, vol. 37, pp. 4318-4323, June 2010.

[12] N. Gordini, "A genetic algorithm approach for SMEs bankruptcy prediction: Empirical evidence from Italy", Expert Systems with Applications, vol. 41, pp. 6433-6445, April 2014.

(C) Geng and Zhang; Licensee Bentham Open.

This is an open access article licensed under the terms of the Creative Commons Attribution Non-Commercial License (http://creativecommons.org/licenses/by-nc/4.0/) which permits unrestricted, non-commercial use, distribution and reproduction in any medium, provided the work is properly cited. 